\title{
Trombolisis en el ictus isquémico agudo en hospitales básicos. A propósito de cuatro casos
}

\author{
A. Varela*, P. de Rojas, R. Vegas, A. Vázquez, B. Cabello y M. Zaheri \\ Servicio de Cuidados Críticos y Urgencias, Hospital de Antequera, Antequera, Málaga, España
}

Recibido el 26 de febrero de 2010; aceptado el 6 de abril de 2010

Disponible en Internet el 20 de mayo de 2010

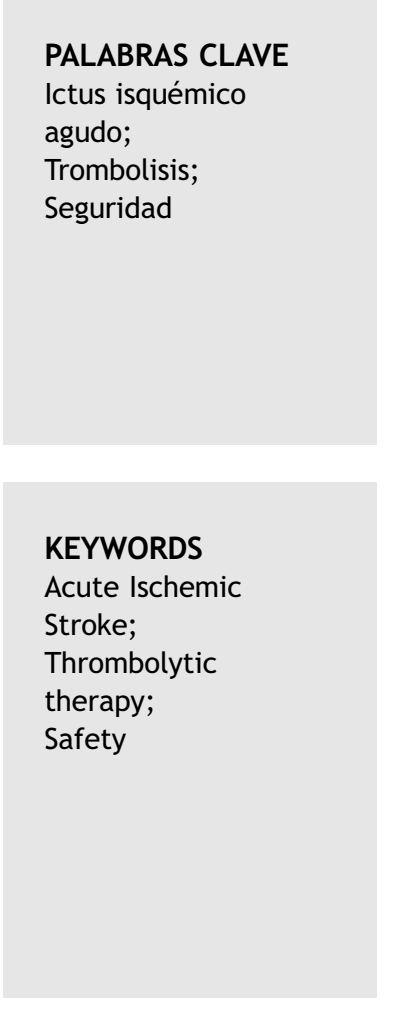

\begin{abstract}
Resumen
El tratamiento trombolítico se ha consolidado en los últimos años como una opción terapéutica efectiva en el ictus isquémico. Existen variadas experiencias en la administración de tratamiento trombolítico en hospitales básicos y rurales. Describimos cuatro casos en los que hemos realizado tratamiento trombolítico en un hospital básico. Hemos utilizado un protocolo estricto de administración basado en el protocolo descrito en el National Institute of Neurological Disorders and Stroke (NINDS) rt-PA stroke study. No hemos detectado ni complicaciones hemorrágicas ni violaciones del protocolo. Concluimos que este protocolo nos ha permitido ofrecer un tratamiento seguro a nuestros pacientes con ictus isquémico.

(c) 2010 Elsevier España, S.L. y SEMICYUC. Todos los derechos reservados.
\end{abstract}

Thrombolysis in acute ischemic stroke in basic hospitals. Four cases reports

\begin{abstract}
Thrombolytic drugs have been used in recent years as an effective therapeutic option in ischemic stroke. There are several experiences with the administration of thrombolytic therapy in basic and rural hospitals. We are describing our experience with four cases in which this treatment was used in our basic hospital during the last three years. We used a strict protocol described in the National Institute of Neurological Disorders and Stroke (NINDS) rt-PA stroke study. No intracranial hemorrhage or protocol violations were detected. We conclude that this protocol has allowed us to offer safe treatment to our ischemic stroke patients.
\end{abstract}

(c) 2010 Elsevier España, S.L. and SEMICYUC. All rights reserved.

\footnotetext{
*Autor para correspondencia.

Correo electrónico: varela.anto@gmail.com (A. Varela).
} 


\section{Introducción}

El accidente cerebrovascular es la primera causa de muerte en España en mujeres y la tercera en varones ${ }^{1}$. Además es especialmente invalidante por las secuelas neuropsicológicas que determina en los pacientes que no fallecen ${ }^{2}$.

El tratamiento trombolítico por vía venosa periférica se ha consolidado en los últimos años como una opción terapéutica efectiva en el ictus isquémico agudo (IIA) ${ }^{3-5}$. La posibilidad de complicaciones secundarias a dicho tratamiento y de elevada morbimortalidad, en concreto la hemorragia cerebral, ha abierto un importante debate en la literatura mundial acerca de la organización necesaria para la administración de dicho tratamiento con una adecuada seguridad $^{6-12}$.

Existen variadas experiencias organizativas que describen la administración de tratamiento trombolítico en hospitales básicos $^{8-13}$. Presentamos nuestra experiencia de los últimos tres años, con cuatro casos en los que hemos realizado tratamiento trombolítico en un hospital comarcal.

\section{Casos clínicos}

Caso 1: mujer de 73 años sin factores de riesgo vascular que acudió a urgencias por presentar cuadro de inicio súbito de disminución de la movilidad en hemicuerpo izquierdo con caída al suelo. A su llegada a urgencias estaba consciente con desviación conjugada de la mirada a la derecha y hemiplejía completa izquierda, hemihipoestesia y reflejo de Babinsky izquierdo presente. Puntuación en la escala de ictus del Instituto Nacional de Salud (National Institute of Health Stroke Scale: NIHSS) de 16. TC craneal sin lesiones evidentes. Tras consentimiento informado escrito se aplicó tratamiento trombolítico a los $150 \mathrm{~min}$ del inicio de los síntomas. En su evolución en las primeras horas se objetivó clara mejoría de la movilidad de hemicuerpo izquierdo, persistiendo al alta hemiparesia izquierda. Se mantuvo en $\mathrm{UCl}$ en las primeras $48 \mathrm{~h}$ para vigilancia neurológica y control de la tensión arterial. Una RNM cerebral a los 10 días de evolución mostró una lesión en región parietal derecha compatible con infarto en territorio de cerebral media derecha. Eco doppler con placas de ateroma calcificados en ambas carótidas comunes sin estenosis.

Se mantuvo hospitalizada en planta durante 13 días donde se inició rehabilitación motora siendo dada de alta con buen resultado funcional, leve hemiparesia residual.

Caso 2: varón de 75 años, dislipemia en tratamiento hipolipemiante como único factor de riesgo cardiovascular. Traído a urgencias por cuadro de hemiparesia izquierda, parálisis facial central izquierda, hipoestesia en hemicuerpo izquierdo, disartria y Babinsky izquierdo. TC cráneo sin alteraciones. NIHSS 19. Tras consentimiento informado escrito se administró tratamiento trombolítico con $150 \mathrm{~min}$ de evolución desde el inicio de los síntomas. Evolución inicial manteniendo semiología neurológica, con TC de control a las $24 \mathrm{~h}$ de la trombolisis con infarto en territorio de arteria cerebral media con edema citotóxico y desviación de línea media. Se añadió tratamiento con manitol, objetivándose progresiva mejoría del nivel de conciencia. Alta a planta a las $36 \mathrm{~h}$ de evolución. Se diagnosticó también neumonía nosocomial con buena evolución con antibioterapia empírica. En RNM practicada el octavo día de evolución se objetivó lesión en región posterior de lóbulo frontal derecho, lóbulos parieto temporal en su totalidad y región anterior de lóbulo occipital, afectación de ganglios de la base respetando el tálamo y signos de herniación subfalciana. El Doppler carotídeo demostró oclusión trombótica completa de la carótida derecha con oclusión del $70 \%$ de carótida izquierda.

Fue alta a domicilio a los 19 días de hospitalización tras normalización del nivel de conciencia y resolución de la neumonía, persistiendo en situación de hemiplejia izquierda, facial central izquierdo y disartria. Se incluyó en programa de rehabilitación.

Caso 3: varón de 54 años fumador y con episodio compatible con accidente isquémico transitorio dos años antes. Trasladado a urgencias del hospital por hemiplejía derecha de dos horas de evolución, objetivándose plejia completa de la extremidad superior y paresia de la extremidad inferior, NIHSS 7. Tras consentimiento informado escrito se realizó trombolisis aproximadamente a los $165 \mathrm{~min}$ del inicio de los síntomas. Exploración neurológica con mejoría progresiva, quedando posteriormente con recuperación casi completa a los $120 \mathrm{~min}$ de la trombolisis, persistiendo solo mínima pérdida de la supinación en extremidad superior derecha al alta. TC de control a las 48 de evolución con pequeño infarto parietal izquierdo. En su evolución en planta se realizó Doppler de troncos supraórticos mostrando pequeña placa de ateroma calcificada de $5 \mathrm{~mm}$ de extensión en carótida interna izquierda. Alta a domicilio tras 7 días de hospitalización.

Caso 4: mujer de 52 años fumadora, sin otro factor de riesgo que fue trasladada a urgencias por hemiplejía derecha y afasia de instauración brusca. Destacó a su ingreso hemiplejía completa derecha y Babinsky derecho, NIHSS de 18. Se realizó TC craneal sin que se objetivaran alteraciones. Tras realizar consentimiento informado se aplicó trombolisis a los $150 \mathrm{~min}$ del inicio de los síntomas. A las $24 \mathrm{~h}$ persistía en la misma situación neurológica, realizando TC de control que mostraba infarto isquémico extenso en territorio de la arteria cerebral media derecha, con edema y efecto masa, por lo que se asoció tratamiento antiedematoso. Pese a ello $48 \mathrm{~h}$ después presentó rápido deterioro neurológico, con nuevo TC mostrando progresión del edema y signos de enclavamiento de tronco, desestimándose cirugía descompresiva. Finalmente la paciente fue incluida en protocolo de donación de órganos.

\section{Discusión}

La efectividad de la trombolisis en el IIA se basa en el hecho de que la revascularización cerebral consigue la recuperación funcional de la zona de cerebro afectada de forma reversible por la isquemia, favoreciendo una mejor recuperación clínica del paciente ${ }^{3}$. Este efecto clínico beneficioso quedó demostrado por un gran ensayo clínico aleatorizado controlado, el National Institute of Neurological Disorders and Stroke (NINDS) rt-PA stroke study, que objetivó una mejor recuperación funcional a los tres meses de los pacientes a los que se administró rt-PA en las primeras tres horas de evolución clínica ${ }^{4}$. 
El hecho de disponer de un tratamiento efectivo para esta patología de tan alta incidencia y morbimortalidad ${ }^{1,2}$ supone un auténtico reto para los sistemas de salud en cuanto a la necesidad de asegurar su administración a los pacientes de forma generalizada y ecuánime y en condiciones de adecuada seguridad $^{7,13}$, ya que por un lado la principal complicación del uso de trombolíticos en el IIA es la hemorragia cerebral sintomática, complicación de alta morbimortalidad y porque por otro el margen temporal de uso dentro de una adecuada relación riesgo/beneficio está limitado a las primeras horas de evolución del proceso. Además debemos recordar que una vez se sienta el diagnóstico de infarto cerebral agudo, la posibilidad de ofertar una reperfusión no debe demorarse, pues los resultados son, al igual que en el infarto agudo de miocardio, tiempo dependiente ${ }^{14}$, hasta el punto que se ha acuñado el aforismo de «tiempo es cerebro».

La posibilidad de asistir a estos pacientes dentro de unidades especializadas de ictus, donde el tratamiento trombolítico se incluye como parte de una atención integral especializada, parece la solución organizativa óptima ${ }^{6}$; sin embargo, en nuestro país el desarrollo de estas unidades queda limitado en gran medida a grandes hospitales. Nuestro hospital básico atiende a una población de 120.000 habitantes, sin que dispongamos de neurólogo. Nuestro hospital de referencia con unidad de ictus se encuentra aproximadamente a una hora de traslado en ambulancia. La necesidad de iniciar el tratamiento trombolítico sin retraso una vez se realiza el diagnóstico y se comprueba la ausencia de contraindicaciones, dentro de las tres primeras horas de evolución, nos ha impulsado a iniciar la administración de este tratamiento tras admitir al paciente en la $\mathrm{UCl}$ del hospital. En nuestro protocolo de actuación la decisión de administrar el tratamiento trombolítico recae en el médico intensivista. Contamos además con la posibilidad de consulta telefónica con el neurólogo en nuestro hospital de referencia. Hemos utilizado el protocolo de aplicación para la trombolisis descrito en el National Institute of Neurological Disorders and Stroke (NINDS) rt-PA Stroke Study ${ }^{4}$.

La indicación actual del tratamiento trombolítico en el IIA se basa en una exploración neurológica sistematizada basada en la adaptación al castellano de la escala NIHSS ${ }^{15}$, una batería analítica inicial, una adecuada exclusión de contraindicaciones y una prueba de imagen, habitualmente TC, que excluya la hemorragia intracraneal. Diferentes autores concluyen que una administración dentro de un protocolo estricto es la mejor garantía de seguridad para el paciente ${ }^{3,4,8-12}$. La aplicación de tratamiento trombolítico en hospitales básicos y dentro de unidades no especializadas ha sido motivo de discusión en cuanto a su seguridad. Se han descrito diferentes estrategias para su administración en estas unidades, desde la valoración remota de pacientes por parte de la unidad de ictus a través de telemedicina, el asesoramiento telefónico o el entrenamiento previo de los médicos del servicio de emergencias ${ }^{7-11}$. En todos los casos se describen porcentajes de complicaciones similares a las descritas en el estudio de referencia, el NINDS ${ }^{4}$, concluyendo que tales estrategias son seguras y efectivas. De los cuatro casos en que aplicamos tratamiento trombolítico, en dos de ellos se constató una evolución favorable con recuperación completa o casi completa al alta hospitalaria. En los otros dos casos no se objetivó mejoría neurológica significativa, falleciendo una paciente por complicaciones del infarto cerebral. No se observaron complicaciones hemorrágicas en ninguno de los casos ni se detectaron violaciones del protocolo.

Aunque con una muestra de tamaño tan reducido no pueden extraerse conclusiones en cuanto al resultado, sí que concluimos que con la dotación actual de un hospital básico de nuestro entorno puede administrarse tratamiento trombolítico en el IIA siguiendo un protocolo de administración de forma estricta, lo cual es una garantía de accesibilidad y seguridad para toda la población respecto a este tratamiento.

\section{Bibliografía}

1. Raziel. Mortalidad por todas las causas. [Base de datos en Internet]. Madrid: Instituto de salud Carlos III. Centro Nacional de Epidemiología; 1980.[consultado 20/1/2010]. Disponible en: http://www.isciii.es/htdocs/centros/epidemiologia/epi_servido res.jsp.

2. An acute need for better stroke care. Lancet Neurol. 2007;6:571.

3. Minematsu K. Recent advances in the acute stroke management. Int Congr Ser. 2006;1290:25-9.

4. The National Institute of Neurological Disorders and Stroke rt-PA Stroke Study Group. Tissue plasminogen activator for acute ischemic stroke. N Engl J Med. 1995;333:1581-7.

5. Navarrete P, Pino F, Rodríguez R, Murillo F, Jiménez MD. Manejo inicial del ictus isquémico agudo. Med Intensiva. 2008;32: 431-43.

6. Masjuan J, Arenillas J, López-Fernández JC, Calleja S. Unidades de ictus: el mejor tratamiento para los pacientes con ictus. Med Intensiva. 2009;33:407.

7. Navarrete P. Réplica a la carta correspondiente al artículo publicado «Manejo inicial del ictus isquémico agudo». Med Intensiva. 2009;33:408.

8. Gross H, Hall C, Switzer JA, Adams RJ, Wang S, Hess DC, et al. Using tPA for acute stroke in a rural setting. Neurology. 2007;68:1957-8.

9. Gebhardt JG, Norris TE. Acute Stroke Care at Rural Hospitals in Idaho: Challenges in Expediting Stroke Care. J Rural Health. 2006;22:88-91.

10. Switzer A, Hall C, Gross H, Waller J, Nichols FT, Wang S, et al. A web-based telestroke system facilitates rapid treatment of acute ischemic stroke patients in rural emergency departments. J Emerg Med. 2009;36:12-8.

11. Lyden PD. Using tPA for acute stroke in a rural setting. Neurology. 2007;648:247.

12. Edwards LL. Using tPA for acute stroke in a rural setting. Neurology. 2007;68:292-4.

13. Camacho JA, Jurado B, Jiménez JM, Montijano AJ, Molina A. Ictus y trombolisis en un hospital básico. Med Intensiva. 2006;30: 238-9.

14. Murillo $\mathrm{F}$, Jiménez $\mathrm{J}$, en representación de los miembros del grupo Proyecto PLACA. Iniciativa multidisciplinaria para la mejora de la atención en el ataque cerebrovascular (ictus) en Andalucía. Proyecto PLACA. Rev Clin Esp. 2005;205:226-9.

15. Montaner J, Álvarez-Sabín J. La escala de ictus del National Institute of Health (NIHSS) y su adaptación al español. Neurologia. 2006;21:192-202. 